\title{
Breast Necrosis Following Coronary by Pass Graft Surgery: Report of Two Cases \& Review of Literature
}

\author{
Maha Abdel Hadi*, Yasir Ghonemi, Mohammed Regal, Saeed Al Shomimi \\ Breast \& Cardiothoracic Surgery Divisions, Department of Surgery, University of Dammam, Dammam, Kingdom of Saudi Arabia \\ Email: ${ }^{*}$ abdelhadi_m@hotmail.com
}

Received September 29, 2013; revised October 29, 2013; accepted November 6, 2013

Copyright (c) 2013 Maha Abdel Hadi et al. This is an open access article distributed under the Creative Commons Attribution License, which permits unrestricted use, distribution, and reproduction in any medium, provided the original work is properly cited.

\begin{abstract}
Breast is an external organ with abundant blood supply which renders it vulnerable to many inflammatory or neoplastic conditions, yet it remains immune to ischemia. Various chest wall surgical procedures may directly or indirectly affect the breast or its overlying skin. Cardiac surgery with its designed incisions is closely related to the breast terrain. Breast necrosis is very rare and only few cases were reported in the literature. We report two cases of breast necrosis in diabetic patients following cardiac bypass surgery. This emerging quandary is an alert to cardiothoracic surgeons to generate special preparation for a subset of patients prior to cardiac surgical procedures in order to minimize the occurrence of ischemia.
\end{abstract}

Keywords: Breast Necrosis; Cardiac Bypass Surgery; Complications

\section{Introduction}

The breast is a vascular organ, gaining its abundant blood supply from the adjacent axillary artery, internal thoracic artery and inter-costal vessels. Fat necrosis and chest wall necrosis are reported entities related to breast trauma, severe infections or chest wall irradiation [1]. However, total breast necrosis is of remote occurrence.

With the current advances in surgical practice, modern operating rooms and specialized anesthesia, coronary artery bypass graft surgery (CABG) has gained momentum over recent years successfully treating the overwhelming increased incidence of coronary heart disease. Harvesting the internal thoracic artery as a graft conduit for myocardial revascularization is a common practice in cardiac surgery [2]. Reported common complications related to this type of surgery are bleeding, heart arrhythmias, and less commonly chest wound infections, memory loss, kidney failure, stroke and heart attacks [3].

To our knowledge, total breast necrosis following CABG surgery is a rare event and has only sporadically been reported in the literature.

\section{Case Reports}

\subsection{Case I}

78-year-old diabetic Saudi female was referred as a case

${ }^{*}$ Corresponding author. of painful left breast mass six weeks following coronary artery bypass graft (CABG) procedure. She was referred as a possible case of breast cancer. She gave history of discharge from the lower end of the sternotomy two weeks after the surgery followed by gaping wound which lasted for four weeks. On local examination, there was a healed sternotomy scar; a tender $2 \times 3 \mathrm{~cm}$ indurated area was appreciated at the left medial lower quadrant. Cord like structure was felt at the affected area with no definite palpable mass. No palpable axillary nodes. The patient underwent investigations in the form of breast ultrasound and CT scan of the chest wall, both were non conclusive however, there was no evidence of a mass or collection. Suggested Mammogram and MRI were deferred since the patient could not tolerate both procedures. Needle aspiration did not reveal any collection; cytology was reported as inflammatory cells with no evidence of malignancy. The mass and local symptoms persisted with no response to antibiotic therapy for six weeks. Localized excision of the affected area revealed no collection or malignancy. Healing was delayed for more than 12 weeks. Follow up at the fifth months of the procedure has shown complete healing of the affected area with marked resolution of the area of induration (Figure 1).

\subsection{Case II}

54-year-old diabetic Saudi female presented to the emer- 


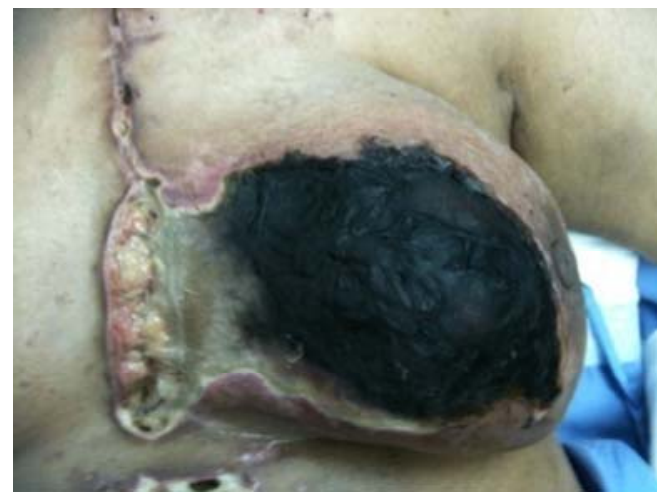

(a)

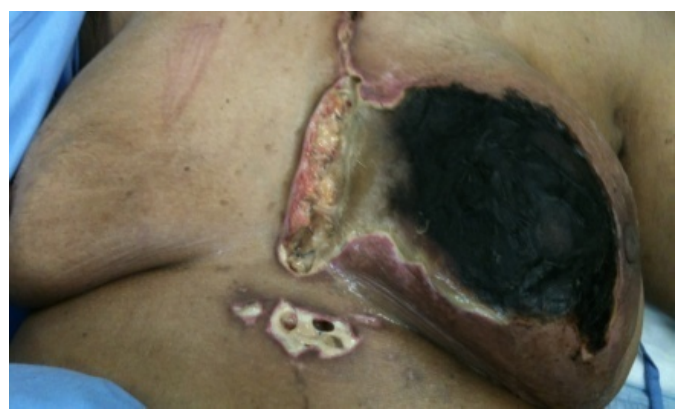

(b)

Figure 1. (a,b) Preoperative status demonstrating total necrosis of the left breast with infected and dehisced sternotomy and drainage wounds.

gency room department three months after CABG procedure with total black discoloration of the left breast. She gave history of progressive discoloration of the breast skin was observed at day 40 of the procedure, accompanied by serous discharge from the lower sternotomy wound. Local examination revealed infected and partially dehisced sternotmy wound, left breast showed dry gangrene of all breast quadrants. CT scan of the chest demonstrated no intra thoracic or chest wall collection.

Emergency breast and chest wall debridement revealed total breast necrosis sparing only the limited skin of the lateral breast quadrants. This patient underwent left breast mastectomy, due to total breast necrosis and extensive chest wall debridement. Despite the aggressive local treatment the wound did not show signs of recovery or healing. The patient had a stormy post operative course and never regained full recovery and expired six weeks after struggle with resistant infection.

Histological analysis from both cases has shown ischemic changes at the periphery of the specimen with extensive tissue necrois and inflammatory cells infiltration. No evidence of calcification or underlying neoplastic changes. No causitive organisms were isolated.

\section{Discussion}

The breast is a vascular organ receiving its abundant blood supply from neighboring arterial network, namely axillary, Internal thoracic and intercostal arteries. Thoracic and cardiac procedures rarely affect the breast cosmesis or its blood supply. Chest wall necrosis due to ischemia is an extremely rare event. Yet, it is seldom reported compared to inflammatory, iatrogenic and self inflicted injuries.

Various external insults or iatrogenic causes of chest wall necrosis have been reported. Extravasation injuries are common as in the case of hydrochloric acid infusion employed for the treatment of metabolic alkalosis which leads to chest wall necrosis [4]. With the increased incidence of early breast cancer detection, breast conserving surgery has gained popularity over recent years. Radiation is an integral part of therapy in breast conservation. Chest wall necrosis following irradiation may also occur [5].

Chest wall necrosis due to self inflicting trauma using variable chemicals or extensive chest wall infections have been reported [6] Drugs such as warfarin has been incriminated in a subset of patients as a cause of skin necrosis and the chest wall is no exception [7].

Internal thoracic artery (ITA) grafts are currently the gold standard for myocardial revascularization. The ITA provides a long conduit that resists atherosclerosis after implantation and offers long-term patency. This vessel is widely used as a conduit; in about 95\% of patients requiring a coronary graft. However, in a small but significant subset of patients both ITAs utilized for revascularization [2].

Other reported vessels used over the past two decades the radial and Inferior Epigastric (IEA) arteries. However, the use of IEA grafts remains limited due to their short length and variation in size and anatomy [8]. Harvesting both ITAs and IEAs can lead to clinically significant ischemia of the anterior abdominal wall [2,3].

They are primarily used as Y grafts from another arterial conduit. Bilateral harvest of ITAs and IEAs results in a moderate risk of clinically significant abdominal wall necrosis. The extent of tissue loss may involve skin, muscle, and fascia, but the peritoneum and posterior rectus sheath are spared and remain intact in both reported cases. These data may be most valuable to those who contemplate an abdominal operation in a patient who has had one or more of their ITAs or IEAs taken [2].

However, complications associated with ITAs only may range from skin dehiscence to complete avascular necrosis of the sternum [9].

Histological analysis of the excised breast tissue demonstrated mural vascular calcifications and intimal proliferation of small sized and medium vessels, a condition known as calciphlaxis, which is thought to cause ischemic necrosis of the skin and septal panniculitis [10].

With the evolving era of minimal invasive surgery and 


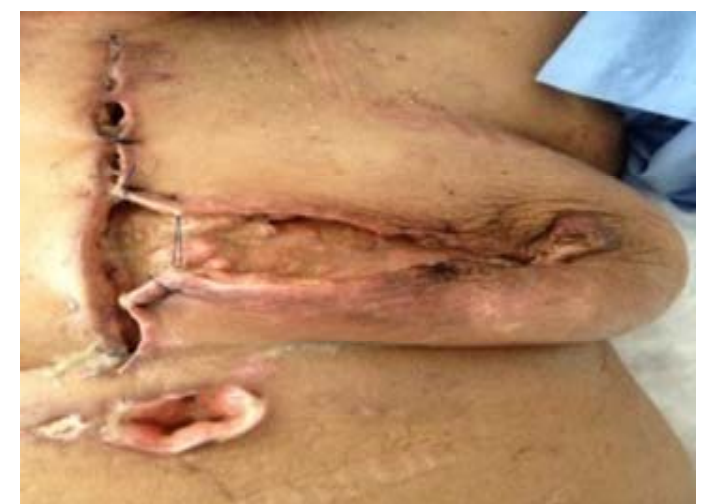

Figure 2. Postoperative status demonstrating the non-healing of the debrided area 6 weeks after the procedure.

the advances of endovascular procedures, we can understandably predict that additional cases of ischemic chest wall necrosis may emerge. The use of covered stents over long sections of the aorta and other major arteries leads to occlusion of smaller arteries at their origins abolishing the potential development of collaterals. In a subset of patients with advanced atherosclerosis undergoing CABG procedure utilizing ITA and (or) IEA, may increase their risk to become candidates to develop large islands of full thickness chest or abdominal wall necrosis large requiring long term and intensive therapy [2].

Predictive factors for patients who are liable to develop chest wall necrosis are not clear cut. Obvious considered risk factors which may promote complications are co-morbid conditions such as diabetes, hypertension, obesity marcromstia, end stage renal disease and calciphlaxis [11-13].

Our two reported cases were patients with uncontrolled diabetes. We feel the early presentation of the first case has contributed to her recovery despite the slow progress. As for the second case, in addition to her pre existing co morbid condition her presentation was 6 weeks post surgery (Figure 2). This certainly had and negative impact on the overall outcome. Considering their underlying co morbid conditions special consideration and precautions may have impacted on their outcomes. Early intervention might have been the key in the success of management.

\section{Conclusion}

In conclusion, the inevitable increased incidence of coronary artery disease and the advances in interventional treatment modalities, increase the risks of atypical complications. Breast necrosis after myocardial revascularization using the ITA is extremely rare. Yet, due to the gravity of the consequences, accurate patient selection and anticipation of precipitated complications will allow better patient preparation and prompt management.

\section{REFERENCES}

[1] A. Bintoudi, T. Malkotsi, F. Goutsaridou, M. Emmanoullidou and I. Tsitouridis, "Breast Necrosis Following Coronary Artery Bypass Grafting,” The Breast Journal, Vol. 17, No. 1, 2011, pp. 83-86.

http://dx.doi.org/10.1111/j.1524-4741.2010.01016.x

[2] D. Johnson, F. Johnson and H. Barner, “Abdominal Wall Necrosis after Harvest of Both Internal Thoracic and Inferior Epigastric Artries," The Annals of Thoracic Surgery, Vol. 91, No. 1, 2011, pp. 38-41.

http://dx.doi.org/10.1016/j.athoracsur.2010.08.060

[3] D. L. Gonyon Jr., M. R. Zenn, C. A. Milano and L. S. Levin, "Breast Necrosis Following Use of the Internal Mammary Artery for Coronary Artery Bypass,” Annals of Plastic Surgery, Vol. 54, No. 1, 2005, pp. 88-91. http://dx.doi.org/10.1097/01.sap.0000141597.38288.3c

[4] I. B. Buchanan, B. T. Campbell, M. D. Peck and B. A. Cairns, "Chest Wall Necrosis and Death Secondary to Hydrochloric Acid Infusion for Metabolic Alkalosis," Southern Medical Journal, Vol. 98, No. 8, 2005, pp. 822824.

http://dx.doi.org/10.1097/01.smj.0000172781.27664.87

[5] A. Yi, H. Kim, H. Jung, M. Huh, S. Ahn and B. Seo, "Radiation-Induced Complications after Breast Cancer Radiation Therapy: A Pictorial Review of Multimodality Imaging Findings,” Korean Journal of Radiology, Vol. 10, No. 5, 2009, pp. 496-507. http://dx.doi.org/10.3348/kjr.2009.10.5.496

[6] M. Eskandarlou and A. H. Moaddab, "Chest Wall Necrosis and Empyema Resulting from Attempting Suicide by Injection of Petroleum into the Pleural Cavity," Emergency Medicine Journal, Vol. 27, No. 8, 2010, pp. 616618. http://dx.doi.org/10.1136/emj.2009.073486

[7] D. Ad-El, A. Meirovitz, A. Weinberg, et al., "Warfarin Skin Necrosis: Local and Systemic Factors," British Journal of Plastic Surgery, Vol. 53, , 2000, pp. 624-626. http://dx.doi.org/10.1054/bjps.2000.3402

[8] K. D. Fong, H. M. Song, T. D. Fang, S. M. Warren and H. P. Lorenz, "Anatomy and Physiology of the Abdominal Wall and Pelvis,” In: G. R. D. Evans, Ed., Reconstructive Surgery of the Chest, Abdomen, and Pelvis, Marcel Dekker, New York, 2004, pp. 325-337. http://dx.doi.org/10.1201/b14208-23

[9] M. S. Wong, J. Kim, C. Yeung and S. H. Williams, "Breast Necrosis Following Left Internal Mammary Artery Harvest: A Case Series and a Comprehensive Review of the Literature,” Annals of Plastic Surgery, Vol. 61, No. 4, 2008, pp. 368-374. http://dx.doi.org/10.1097/SAP.0b013e3181640851

[10] D. J. Morris, A. H. Fischer and J. Abboud, "Breast Infarction after Internal Mammary Artery Harvest in a Patient with Calciphylaxis," The Annals of Thoracic Surgery, Vol. 64, No. 5, 1997, pp. 1469-1471. http://dx.doi.org/10.1016/S0003-4975(97)00923-5

[11] G. Speziale, G. Ruvolo and B. Marino, "Flow Capacity of Inferior Epigastric Artery in Composite Arterial Grafts,” The Journal of Cardiovascular Surgery (Torino), Vol. 40, No. 6, 1999, pp. 857-859. 
[12] K. Cathenis, D. Goossens, R. Vertriest, M. Coppens and R. Hamerlijnck, "Breast Infarction Due to Calciphylaxis after Coronary Artery Bypass Grafting," The Annals of Thoracic Surgery, Vol. 91, No. 5, 2011, pp. 1603-1606. http://dx.doi.org/10.1016/j.athoracsur.2010.10.088
[13] A. Rashid, M. Haj Basheer and K. Khan, "Breast Necrosis Following Harvest of Internal Mammary Artery," British Journal of Plastic Surgery, Vol. 57, No. 4, 2004 pp. 366-368. http://dx.doi.org/10.1016/j.bjps.2004.01.006 